\title{
Dimensional evolution between one- and two-dimensional topological phases
}

\author{
Huaiming Guo* and Yang Lin \\ Department of Physics and Spintronics Interdisciplinary Center, Beihang University, Beijing, 100191, China
}

Shun-Qing Shen

Department of Physics, The University of Hong Kong, Pokfulam Road, Hong Kong

(Received 16 May 2014; revised manuscript received 9 July 2014; published 12 August 2014)

\begin{abstract}
Dimensional evolution between one- (1D) and two-dimensional (2D) topological phases is investigated systematically. The crossover from a $2 \mathrm{D}$ topological insulator to its $1 \mathrm{D}$ limit shows oscillating behavior between a 1D ordinary insulator and a 1D topological insulator. By constructing a 2D topological system from a 1D topological insulator, it is shown that there exist possibly weak topological phases in 2D time-reversal invariant band insulators, one of which can be realized in anisotropic systems. The topological invariant of the phase is $Z_{2}=0$. However, the edge states may appear along specific boundaries. It can be interpreted as arranged 1D topological phases, and have a symmetry-protecting nature as does the corresponding 1D topological phase. Robust edge states can exist under specific conditions. These results provide further understanding of 2D time-reversal invariant insulators, and can be realized experimentally.
\end{abstract}

DOI: 10.1103/PhysRevB.90.085413

PACS number(s): 73.43.-f, 03.65.Vf, 73.20.-r

\section{INTRODUCTION}

A topological insulator (TI) is a novel quantum state of matter, which is determined by the topological properties of its band structure. It has generated great interest in the field of condensed matter physics and material science due to its many exotic electromagnetic properties and possible potential applications [1-4]. Its discovery also deepens the understanding of the time-reversal invariant band insulators. In two dimensions, ordinary insulators and quantum spin Hall insulators are characterized by a $Z_{2}$ invariant $v: v=0$ for a conventional insulator and $v=1$ for a quantum spin Hall insulator [5,6]. In three dimensions (3D) time-reversal invariant band insulators can be classified into 16 topological classes distinguished by four $Z_{2}$ topological invariants, and thus the ordinary insulator is distinguished from "weak" and "strong" TIs $[7,8]$.

The 3D TIs have been proposed and verified in many materials [9-13]. However, the 2D TIs have only been realized in $\mathrm{HgTe} / \mathrm{CdTe}$ and $\mathrm{InAs} / \mathrm{GaSb} / \mathrm{AlSb}$ quantum well systems [14-16]. Theoretical studies have suggested that the 2D TI may be achieved in a thin film of 3D TI. In the thin film, the quantum tunneling between the two surfaces generates a hybridized gap at the Dirac point. Depending on the thickness of the quantum wells, the system oscillates between an ordinary insulator and quantum spin Hall insulator $[17,18]$. Typical TI materials, such as $\mathrm{Bi}_{2} \mathrm{Te}_{3}$ and $\mathrm{Bi}_{2} \mathrm{Se}_{3}$ have a layered structure consisting of weakly coupled quintuple layers, which makes it relatively easy to grow high quality crystalline thin films using molecular beam epitaxy. Until now the thin films of 3D TIs have been successfully fabricated experimentally and the gap opening has been observed $[19,20]$. This paves the way to realize the quantum spin Hall insulator from the present various 3D TIs, which will greatly enlarge the family of 2D TIs. Furthermore by introducing ferromagnetism in thin film, the quantum anomalous Hall effect can be realized, which has

\footnotetext{
*hmguo@buaa.edu.cn
}

been experimentally confirmed in magnetic TIs of $\mathrm{Cr}$-doped $(\mathrm{Bi}, \mathrm{Sb})_{2} \mathrm{Te}_{3}[21,22]$. Also it has been shown that the Chern number of quantum anomalous Hall effect can be higher than 1 by tuning exchange field or sample thickness [23,24]. Compared to the 3D strong TIs, the weak TIs are related to the topological property of the lower dimensions in a more direct way, since it can be interpreted as a layered 2D quantum spin Hall insulator. Though no weak TIs have been reported experimentally, they are expected to have interesting physical properties [25-29]. Besides the studies on the 2D limit of 3D TIs, recently a theoretical formalism has been developed to show that a 3D TI can be designed artificially via stacking 2D layers [30]. It provides controllable approach to engineer 'homemade' TIs and overcomes the limitation imposed by bulk crystal geometry.

The above studies show the connection between the 2D and 3D TIs. It is notable that recently there are increasing interests in 1D topological phases [31-34]. Specially they have been studied experimentally using ultra-cold fermions trapped in the optical superlattice and photons in photonic quasicrystals [35,36] and metamaterials [37]. With the developments of these techniques, various 1D models with topological properties may be realized [38]. Also these techniques can be easily extended to $2 \mathrm{D}$ or $3 \mathrm{D}$ cases. It is also desirable to study the $1 \mathrm{D}$ topological phase in real materials. A natural thought is to narrow a 2D TI and the narrow strip may be a 1D TI. Also a formalism on how to construct 2D TIs from 1D ones is needed. The underlying question is the connections between the $1 \mathrm{D}$ and 2D TIs.

In this paper, the question is studied systematically. It is found that the crossover from the 2D quantum spin Hall insulator to its 1D limit shows oscillatory behavior between a 1D ordinary insulator and a 1D TI. Generally the 2D TI is a "genuine" $2 \mathrm{D}$ phase and cannot be understood simply from the corresponding 1D phases. However, by arranging 1D TIs, it is found that there exists a $2 \mathrm{D}$ weak topological phase in anisotropic systems. In contrast to the quantum spin Hall insulator, the 2D weak TI has topological invariant $Z_{2}=0$, and the edge states are midgap ones and only appear 
along specific boundaries. These results provide further understanding of 2D time-reversal invariant insulators, and can be realized experimentally. The paper is organized as follows: In Sec. II, the model Hamiltonian is introduced to describe the 1D and 2D TIs; in Sec. III, an oscillatory crossover from 2D to $1 \mathrm{D}$ topological phases is observed; in Sec. IV, a 2D weak TI is identified by arranging 1D topological models; in Sec. $\mathrm{V}$, the physical properties of the 2D weak TI are studied; and finally, Sec. VI is the conclusion of the present paper.

\section{THE 1D AND 2D TI MODELS}

The starting point of the present work is the 2D tightbinding model for a quantum spin Hall insulator [14,39],

$$
\begin{aligned}
H_{2 \mathrm{D}}= & \sum_{i}(M+4 B) \Psi_{i}^{\dagger} I \otimes \sigma_{z} \Psi_{i} \\
& -\sum_{i, \hat{x}} B \Psi_{i}^{\dagger} I \otimes \sigma_{z} \Psi_{i+\hat{x}}-\sum_{i, \hat{y}} B \Psi_{i}^{\dagger} I \otimes \sigma_{z} \Psi_{i+\hat{y}} \\
& -\sum_{i, \hat{x}} \operatorname{sgn}(\hat{x}) i A \Psi_{i}^{\dagger} s_{z} \otimes \sigma_{x} \Psi_{i+\hat{x}} \\
& -\sum_{i, \hat{y}} \operatorname{sgn}(\hat{y}) i A \Psi_{i}^{\dagger} I \otimes \sigma_{y} \Psi_{i+\hat{y}},
\end{aligned}
$$

where $I$ is the identity matrix and $\sigma_{j}, s_{j}(j=x, y, z)$ are the Pauli matrices representing the orbit and spin, respectively; $\Psi_{i}=\left(s_{i \uparrow}, p_{i \uparrow}, s_{i \downarrow}, p_{i, \downarrow}\right)^{T}$ with $s_{i \uparrow(\downarrow)}\left(p_{i \uparrow(\downarrow)}\right)$ electron annihilating operator at site $\mathbf{r}_{i}$. The first term is the on-site potential, which has different signs for the $s$ orbit and $p$ orbit. The second and third terms are the hopping amplitudes among the $s$ orbits or $p$ orbits, which differ by a sign. The third and fourth terms are the hopping amplitudes between the $s$-orbit and $p$-orbit electrons, which is due to the spin-orbit coupling. $A$ and $B$ are the hopping amplitudes and in the following, we take $B$ positive and set $A=1$ as a unit of the energy scale. The Hamiltonian is invariant under time reversal $\mathcal{T}=i s_{y} \otimes I \mathcal{K}$. It belongs to the AII class and its topological property is described by a $Z_{2}$ index [40]. For $M>0, M<-8 B$ it is a trivial insulator (Fig. 1). For $-8 B<M<0$ it is a quantum spin Hall insulator with the topological invariant $Z_{2}=1$. In the Hamiltonian, the subsystems of spin up and down are decoupled.

Based on the spin-up subsystem and reducing one dimension (such as the $y$ dimension), a 1D spinless topological model is obtained [32-34]:

$$
\begin{aligned}
H_{1 \mathrm{D}}= & \sum_{i}(M+4 B) \Psi_{i \uparrow}^{\dagger} \sigma_{z} \Psi_{i \uparrow}-\sum_{i, \hat{x}} B \Psi_{i \uparrow}^{\dagger} \sigma_{z} \Psi_{i+\hat{x} \uparrow} \\
& -\sum_{i, \hat{x}} \operatorname{sgn}(\hat{x}) i A \Psi_{i \uparrow}^{\dagger} \sigma_{x} \Psi_{i+\hat{x} \uparrow} .
\end{aligned}
$$

At half-filling, the system is a nontrivial insulator for $-6 B<$ $M<-2 B$ and a trivial insulator for $M>-2 B$ or $M<-6 B$. In the momentum space it becomes $H_{1 \mathrm{D}}(k)=[M+4 B-$ $2 B \cos (k)] \sigma_{z}+2 A \sin (k) \sigma_{x}$. The Hamiltonian possesses a particle-hole symmetry $\sigma_{x} H_{1 \mathrm{D}}^{*}(k) \sigma_{x}=-H_{1 \mathrm{D}}(-k)$, a pseudotime-reversal symmetry $\sigma_{z} H_{1 \mathrm{D}}^{*}(k) \sigma_{z}=H_{1 \mathrm{D}}(-k)$ and a chiral symmetry $\sigma_{y} H_{1 \mathrm{D}}(k) \sigma_{y}=-H_{1 \mathrm{D}}(k)$. It belongs to the BDI class and its topological invariant is a winding number $v$, (a)

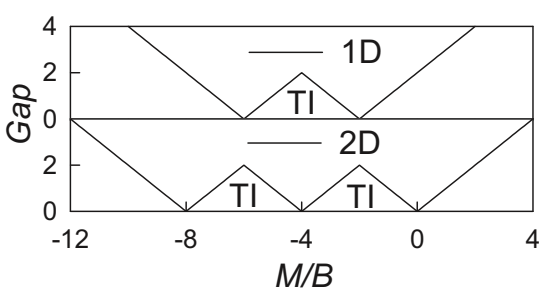

(b)

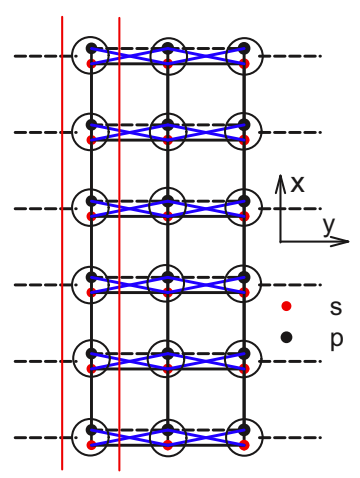

(c)

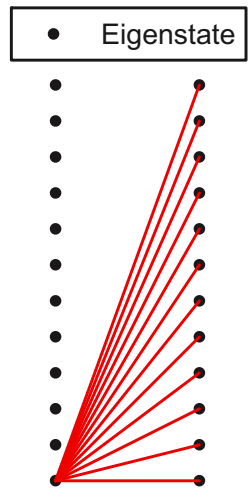

FIG. 1. (Color online) (a) The phase diagram of the Hamiltonian equations (1) and (2). (b) The schematic diagram of the 2D model, Eq. (1), in which the $A$-type (blue solid lines) and $B$-type (dashed and solid black lines) hoppings along the $y$ direction are shown. (c) The real hopping between the NN chains is converted to the hopping between the eigenstates of the NN chains.

which is an integer [41-43]. The winding number of the Hamiltonian equation (2) is $v=1$. In this case, it is equivalent to the Berry phase, which describes the electric polarization. The Berry phase in the $k$ space is defined as $\gamma=\oint \mathcal{A}(k) d k$ with the Berry connection $\mathcal{A}(k)=i\left\langle u_{k}\left|\frac{d}{d k}\right| u_{k}\right\rangle$ and $\left|u_{k}\right\rangle$ the occupied Bloch states $[44,45]$. Due to the protection of the symmetries in the BDI class the Berry phase $\gamma \bmod 2 \pi$ can have two values: $\pi$ for a topologically nontrivial phase and 0 for a topologically trivial phase. The topological property is manifested by the boundary states of zero energy on an open chain. The spin-down subsystem, which is the time-reversal counterpart of Eq. (2), has a winding number $v=-1$. Then the combined system is time-reversal invariant [the time-reversal operator $\mathcal{T}$ is the same as that for Eq. (1)]. Then the combined system belongs to the DIII class and its topological invariant is a $Z_{2}$. The $Z_{2}$ topological invariant for the $s_{z}$ conserved system can be calculated using the Berry phase of either spin subsystem.

\section{THE OSCILLATORY CROSSOVER FROM 2D TO 1D TOPOLOGICAL PHASES}

We consider the 2D TI model, Eq. (1), in a narrow strip configuration. Its finite-size effect has been studied previously [46]. It is found that on a narrow strip the edge states on the two sides can couple together to produce a gap in the spectrum. The finite-size gap $\Delta \propto e^{-\lambda L}$, decays in an exponential law with the width $L$. The decaying length scale is determined by the bulk gap of Eq. (1). As shown in Fig. 2, the bulk gap vanishes at $M / B=0,-4,-8$, near which the finite-size gap is maximum. Interestingly the narrow strip as a quasi1D system shows $1 \mathrm{D}$ topological phase in an oscillatory way. 

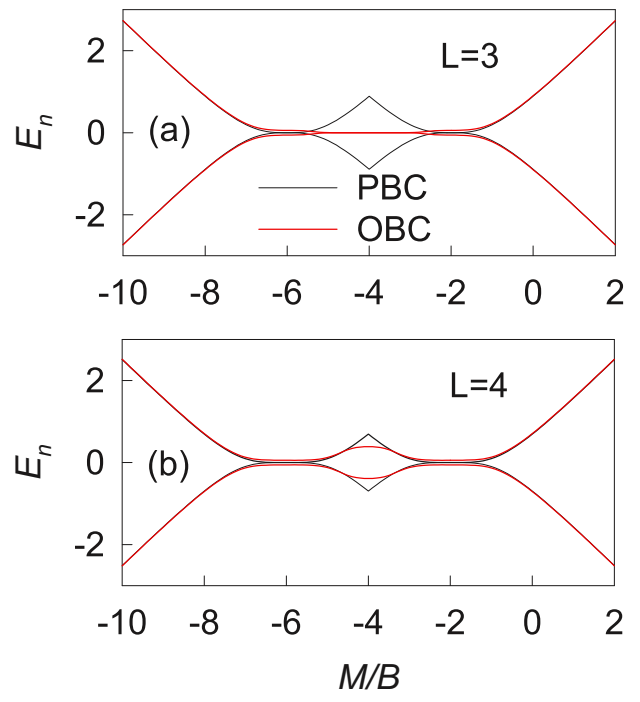

FIG. 2. (Color online) The eigenenergies at half-filling and the one above of Eq. (1) vs $M$ on a thin strip with the width: (a) $L=3$; (b) $L=4$. The length of the strip is $N=50$ with periodic boundary condition (PBC) or open boundary condition (OBC).

It is a 1D topological phase with boundary states when the width $L$ is odd, while trivial when the width $L$ is even. Since spin $s_{z}$ is conserved in Eq. (1), the oscillatory crossover happens for each spin subsystem. If the spins are coupled (such as by the Rashba spin-orbit coupling described in Sec. V), the above results still persist.

The oscillatory behavior happens near $M / B=-4$, where the bulk gap is zero and separates two TI phases. However, it is absent near $M / B=0,-8$, which separate a TI from a trivial insulator. It is noted in Fig. 1 that $M / B=-4$ is deep in the 1D topological phase of Eq. (2), which is reduced from Eq. (1). So the oscillatory behavior is closely related to the corresponding 1D topological phase. In the following section, we construct the 2D TI model, Eq. (1), from the point of view of coupled 1D models, Eq. (2), to understand the oscillatory behavior.

\section{CONSTRUCTING A 2D MODEL FROM A 1D TOPOLOGICAL MODEL}

The 2D model in Eq. (1) can be viewed as a set of the coupled 1D models in Eq. (2). In the limit of zero coupling, the boundary states of the 1D topological model form two flat bands at the two edges of the 2D system, which are topologically protected by the symmetry of the isolated 1D model. Next we study the evolution of the boundary states as the 1D chains are coupled by the hopping terms. Suppose the Hamiltonian of a 1D open chain along the $x$ direction is $H_{1 \mathrm{D}}^{\text {open, } n}$ [the same as that in Eq. (2)] with $n$ denoting the $n$th chain (it is identical for different $n$ ). Since the Hamiltonian $H_{1 \mathrm{D}}^{\text {open, } n}$ has the chiral symmetry, its eigenenergies are symmetric to 0 and we label them $\{E\}_{n}=-E_{0}^{(n)}, E_{0}^{(n)},-E_{1}^{(n)}, E_{1}^{(n)}, \ldots\left(E_{0}^{(n)}<E_{1}^{(n)} \ldots\right)$, which correspond to the eigenstates $\{\varphi\}_{n}=\varphi_{0}^{n,-}, \varphi_{0}^{n,+}, \varphi_{1}^{n,-}, \varphi_{1}^{n,+}, \ldots$ . For the 2D system containing $N_{y}$ 1D chains, we choose the basis $\Phi=\left(\{\varphi\}_{0},\{\varphi\}_{1}, \ldots,\{\varphi\}_{N_{y}}\right)$. Under this basis, the 2D
Hamiltonian can be calculated and has the following structure:

$$
H_{2 \mathrm{D}}^{\left(N_{y}\right)}=\left(\begin{array}{cccc}
H_{\text {diag }} & H_{\text {ndiag }} & 0 & \ldots \\
H_{\text {ndiag }}^{\dagger} & H_{\text {diag }} & H_{\text {ndiag }} & \ldots \\
0 & H_{\text {ndiag }}^{\dagger} & H_{\text {diag }} & \ldots \\
0 & 0 & H_{\text {diag }} & \ldots
\end{array}\right),
$$

where $H_{\text {diag }}=\left\langle\{\varphi\}_{n}\left|H_{1 \mathrm{D}}^{\text {open }, n}\right|\{\varphi\}_{n}\right\rangle$ is the diagonal matrix with the diagonal elements $\{E\}_{n} ; H_{\text {ndiag }}=\left\langle\{\varphi\}_{n}\left|H_{\text {couple }}\right|\{\varphi\}_{n+1}\right\rangle$ with $H_{\text {couple }}$ the coupling between nearest-neighbor (NN) chains. $H_{\text {ndiag }}$ contains the hopping amplitudes of the eigenstates between the NN chains, and generally one eigenstate couples with all other eigenstates of the NN chain.

We first consider a special case $M=-4 B$, when the zero modes $\left(E_{0}^{(n)}=0\right)$ of the 1D open chain distribute only on the end sites. Each chain has two zero modes, one of which is on one end and the other is on the other end. When only the $A$-type hoppings with the amplitude $A$ couple the chains, the zero modes only couple the zero modes on the same end of the NN chain and the amplitude is $\pm I A$. So the low-energy physics is described by the zero modes which hop on the edge with the amplitude $\pm I A$.

For the case of two chains, we have two zero modes on each side. We can limit to a subspace composed of the zero modes, i.e., $\left(\varphi_{0}^{1,-}, \varphi_{0}^{2,-}\right)$. Under this basis, the effective Hamiltonian becomes a $2 \times 2$ matrix:

$$
H_{e f f}^{(2)}=\left(\begin{array}{cc}
0 & i A \\
-i A & 0
\end{array}\right) .
$$

Its eigenenergy is $E_{e f f}^{(2)}= \pm A$ and the system is gapped. Similarly for the case of three chains, under the basis $\left(\varphi_{0}^{1,-}, \varphi_{0}^{2,-}, \varphi_{0}^{3,-}\right)^{T}$, the effective Hamiltonian becomes a $3 \times 3$ matrix:

$$
H_{e f f}^{(3)}=\left(\begin{array}{ccc}
0 & i A & 0 \\
-i A & 0 & i A \\
0 & -i A & 0
\end{array}\right) .
$$

This matrix has an eigenenergy 0 with the eigenvector $\psi_{0}=$ $\frac{1}{\sqrt{2}}\left(\varphi_{0}^{1,-}+\varphi_{0}^{2,-}\right)$. For the case of multichains, the resulting effective matrix has a similar structure, i.e., a tridiagonal matrix with zero diagonal elements. The eigenenergy of such Hermitian matrix is symmetric to 0 . So if the dimension of the matrix is odd, it must have the eigenvalue 0 . The above result can be understood qualitatively: Since two coupled boundary states tend to destroy each other, one can survive from an odd number of boundary states.

The above result persists when finite $B$-type hopping is included. The $B$-type hopping couples the zero modes with a few other modes. For relatively small $B$, the coupling among the zero modes still dominates. A proper unitary transformation can move the few coupled modes to one side of the matrix, and the zero modes to the other side. If the length of the chain is long enough, i.e., the dimension of the matrix is big enough, their effect on the zero modes can be neglected, which is known as the finite-size effect. So the oscillating behavior of the topological property of the quasi-1D strip created by narrowing a $2 \mathrm{D}$ TI can be understood in the above way.

In a general case of $N_{y}$, the zero modes form a 1D conducting chain and its energy spectrum is $E=2 A \sin \left(k_{y}\right)$ 


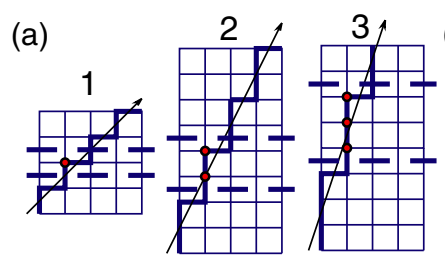

(c)

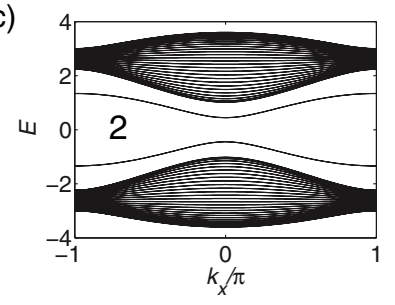

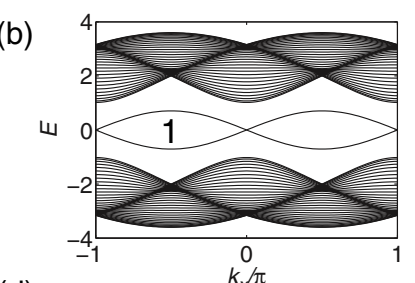

(d)

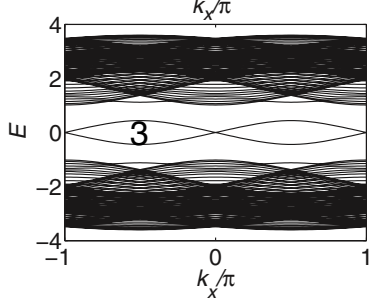

FIG. 3. (Color online) One-dimensional energy bands for a strip with different edges. The parameters are $M=-3 B$ and $B^{\prime}=0$, when the system is in the WTI 1 phase.

with $k_{y}=2 \pi / N_{y}$ (the open boundary condition along the $x$ direction and the periodic boundary condition along the $y$ direction). The edge state is a midgap state. Specifically, the above edge states appear on the edge along the $y$ direction, but disappear on the edge along the $x$ direction. For other kinds of edges, they can be understood from the view of coupling thin strips. As an example, we consider there different edges shown in Fig. 3(a). There are midgap edge states on edges 1 and 3, but not on edge 2. The thin strip in the $x$ direction is shown by the dashed lines in the figure. For the system with edge 2, the thin strip contains two chains. As discussed in the previous section, the boundary modes are gapped, thus there are no midgap edge states when the thin strips are coupled along the $y$ direction. While for the system with edges 1 or 3, the thin strip contains an odd number of chains. So the boundary modes persist and there are midgap edge states.

Since the bulk system is gapped, the appearance of the midgap edge states on specific boundaries is due to the topological property of the bulk insulator. However, it is in contrast to the topological property of quantum spin Hall insulators, where there are always gapless edge states traversing the gap. The topological invariant of the above bulk system is $Z_{2}=0$, which is trivial. However, the above phase is different from a trivial insulator. This implies that for 2D time-reversal invariant insulators besides the trivial insulators and the quantum spin Hall insulators, there exist other insulators, in which $Z_{2}=0$, but the midgap edge states appear on specific boundaries. It is somehow similar to the 3D weak TI phase, which can be understood as layered quantum spin Hall effect. So we term the above phase as "2D weak TI."

\section{THE 2D WEAK TI}

In the previous section a weak 2D TI is identified with the midgap edge states along specific edges and the topological invariant $Z_{2}=0$. More generally the Hamiltonian in Eq. (1) can be modified by changing the amplitude of the $B$-type hopping along the $y$ direction to $B^{\prime}$ which can be tuned. With the points on which the gap closes, the phase diagram of the modified Hamiltonian in the $\left(M, B^{\prime}\right)$ plane can be obtained.

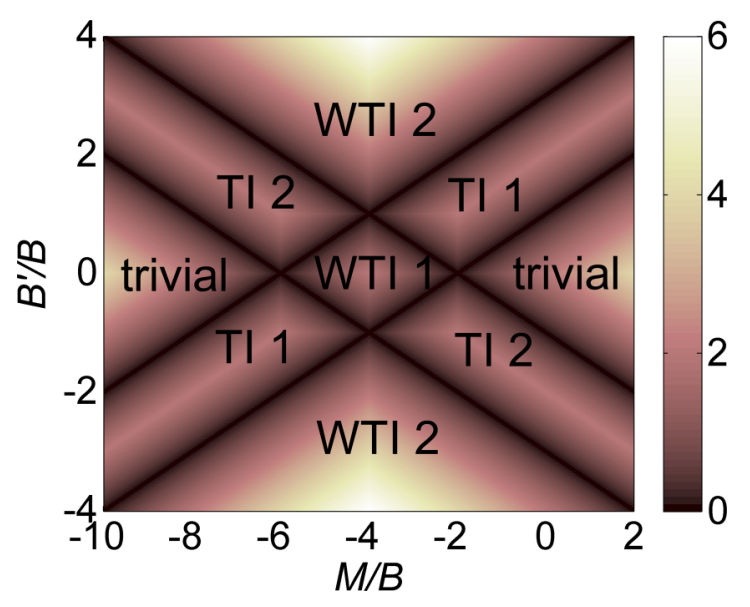

FIG. 4. (Color online) The phase diagram of the modified Hamiltonian equation (1) with anisotropic $B$-type hoppings in the $\left(M, B^{\prime}\right)$ plane. In TI 1 (TI 2) the crossing of the edge states is at $k_{y}=0(\pi)$ with $y$-directed boundary. The color represents the gap of the bulk system.

As shown in Fig. 4, besides the quantum spin Hall insulators and trivial insulators, the 2D weak TI exists in three regions of the phase diagram. We distinguish the 2D weak TIs with the midgap edge states appearing on the $x$ edge (WTI 2) or $y$ edge (WTI 1), and the quantum spin Hall insulators with the gapless crossing appearing at $k_{y}=0$ (TI 1) or $k_{y}=\pi$ (TI 2).

It is noticed that the $2 \mathrm{D}$ weak TI exists in the regions with anisotropic $B$-type hoppings $\left(B^{\prime} \neq B\right)$. Indeed the anisotropy is key to realize the phase [47]. The anisotropy can also be induced in the parameter $M$ of Eq. (1). Consider the case shown in Fig. 5: The mass $M$ is uniform along the $y$ direction, but has alternating values $M_{1}, M_{2}$ along the $x$ direction. The phase diagram in the $\left(M_{1}, M_{2}\right)$ plane is shown in Fig. 5 , in which the $2 \mathrm{D}$ weak TI is identified in six regions.

Till now the 2D weak TI is identified in the anisotropic systems. Its topological invariant $Z_{2}$ is zero, but the phase is different from the $Z_{2}=0$ trivial insulators. It is desirable to

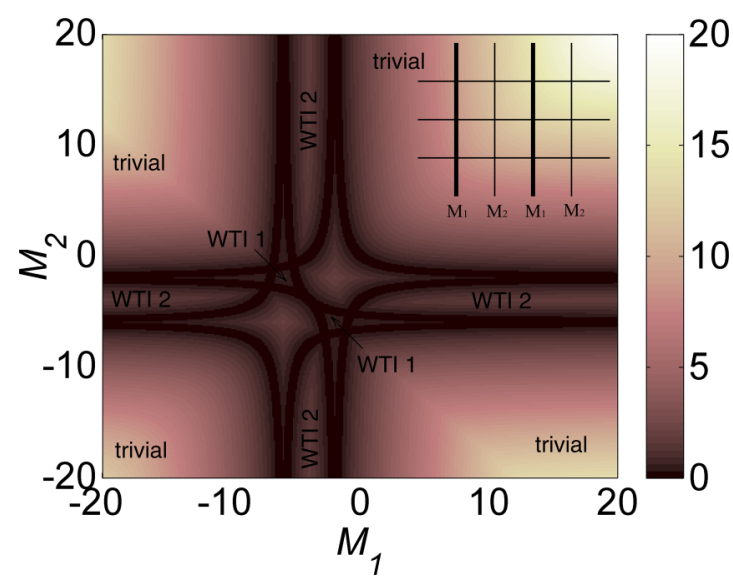

FIG. 5. (Color online) The phase diagram of the modified Hamiltonian equation (1) with anisotropic mass in the $\left(M_{1}, M_{2}\right)$ plane. The inset schematically shows the pattern of the mass on the lattice. The regions without notation represent the quantum spin Hall insulator. The color represents the gap of the bulk system. 
characterize its topological property. It has been known that the $Z_{2}$ topological invariant of a $2 \mathrm{D}$ time-reversal invariant insulator is defined as $(-1)^{\nu_{0}}=\prod_{n_{j}=0,1} \delta_{n_{1} n_{2}}$, with $\delta_{n_{1} n_{2}}$ the time-reversal polarization at the four time-reversal invariant momenta $\Gamma_{i=\left(n_{1} n_{2}\right)}=\left(n_{1} \pi \hat{x}+n_{2} \pi \hat{y}\right)$, with $n_{j}=0,1$. The above constructed $2 \mathrm{D}$ Hamiltonian has the inversion symmetry $H(-\mathbf{k})=\hat{P} H(\mathbf{k}) \hat{P}$ with $\hat{P}$ the inversion operator. In the presence of the inversion symmetry, $\delta_{i}$ can be determined by the parity of the occupied band eigenstates: $\delta_{i}=\prod_{m=1}^{N} \xi_{2 m}\left(\Gamma_{i}\right)$, where $\xi_{2 m}$ is the parity eigenvalues of the $2 m$ th occupied states. The topological property of $2 \mathrm{D}$ time-reversal invariant insulators is determined by four time-reversal polarizations $\delta_{i}$. In general $\delta_{i}$ is not gauge invariant, while in the presence of inversion symmetry $\delta_{i}$ is gauge invariant. So for the $Z_{2}=0$ time-reversal invariant insulators with inversion symmetry, the details of the four $\delta_{i}$ should distinguish the 2D weak TIs and the trivial insulators.

For the case of anisotropic $B$-type hoppings, the inversion operator is $\hat{P}=I \otimes \sigma_{Z}$ and $\delta_{i}=-\operatorname{sgn}\left[M\left(\Gamma_{i}\right)\right]$ with $\tilde{M}(\mathbf{k})=M+4 B-2 B \cos \left(k_{x}\right)-2 B^{\prime} \cos \left(k_{y}\right)$. In WTI 1 , if the boundary is along the $y$ direction, the two $\delta_{i}$ projected on $k_{y}=0(\pi)$ have different signs, which is in contrast to the trivial insulator. Define the $Z_{2}$ invariant $\pi_{k_{\mu}}$ the product of two $\delta_{i}$ on the line $k_{\mu}\left(k_{\mu}=n_{i} \pi, \mu=x, y\right)$, then the additional $Z_{2}$ indices $\pi_{k_{\mu}}$ distinguish the 2D weak TI and trivial insulators. $\pi_{k_{\mu}}$ is directly related to the existence of the edge states on the $\mu$-directed boundary and $\pi_{k_{\mu}}=-1$ means a crossing of the edge states at $k_{\mu}$. For example in the WTI 1 phase shown in Fig. 6(a), if the boundary is along the $y$ direction, $k_{y}$ remains a good quantum number and $\pi_{k_{y}=0}=\pi_{k_{y}=\pi}=-1$. So there appear midgap edge states with two crossings at $k_{y}=0, \pi$ on the boundaries. Also in quantum spin Hall insulators, $\pi_{k_{\mu}}$ determines the position of the crossing of the edge states, which happens at $k_{\mu}$ with $\pi_{k_{\mu}}=-1$.

For the case of anisotropic mass, the inversion operator is $\hat{P}=I \otimes \operatorname{diag}\left(\sigma_{z}, e^{-i k_{y} / 2} \sigma_{z}\right)$ (the inversion center is chosen on a site with the mass $M_{1}$ ). The $\delta_{i}$ of the $2 \mathrm{D}$ weak TI phases are calculated, which is shown in Fig. 6. It seems that the above discussion is inapplicable. Actually to characterize

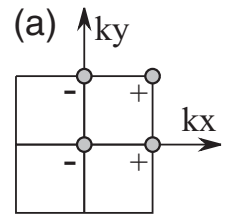

WTI 1
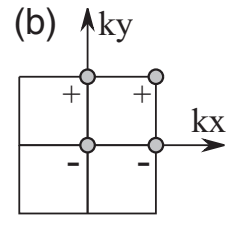

WTI 2 (c) $\uparrow \mathrm{ky}$

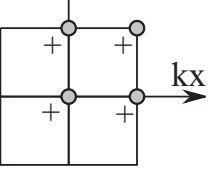

trivial (d) $\uparrow \mathrm{ky}$

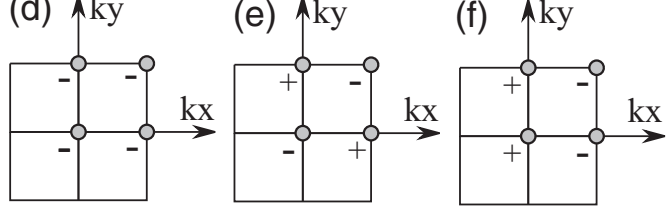

FIG. 6. Depicts $\delta_{i}$ at the time-reversal invariant momenta of 2D weak TIs and trivial insulators. The upper ones are for the case of anisotropic $B$-type hoppings: (a) WTI 1; (b) WTI 2; (c) trivial insulator. The lower ones are for the case of anisotropic mass: (d) WTI 1; (e) WTI 2; (f) trivial insulator.


FIG. 7. (Color online) (a) The zero-gap line in the $\left(M_{1}, M_{2}\right)$ plane for the case of anisotropic mass at the four time-reversal invariant momenta. $+/-$ is the parity of the occupied band. (b) The phase diagram obtained by combining the zero-gap lines in (a). The arrow dashed line 1 (2) is the path to WTI 1 (2) [those corresponding to (d) and (e) in Fig. 6] from a trivial insulator.

the topological property properly, the $\delta_{i}$ should be defined compared to the corresponding $\delta_{i}^{\text {trivial }}$ of the trivial insulator in the same model, i.e., $\tilde{\delta}_{i}=\delta_{i} \delta_{i}^{\text {trivial }}$. With $\tilde{\delta_{i}}$, Fig. $6(\mathrm{~d})$ [6(e)] is the same as Fig. 6(a) [6(b)], and the 2D weak TI is characterized correctly.

So to correctly characterize the $2 \mathrm{D}$ weak TI with inversion symmetry, the $\delta_{i}$ should be defined compared to that of the trivial insulator in the same model. It can be understood from the view of band inverting. We take the case of anisotropic mass as an example. Its Hamiltonian in the momentum space can be written as

$$
H_{M}(\mathbf{k})=\left(\begin{array}{cc}
h_{1}\left(k_{y}\right) & h_{12}\left(k_{x}\right) \\
h_{12}^{\dagger}\left(k_{x}\right) & h_{2}\left(k_{y}\right)
\end{array}\right),
$$

where $\quad h_{1,2}\left(k_{y}\right)=\left[M_{1,2}+4 B-2 B \cos \left(k_{y}\right)\right] \sigma_{z}+$ $2 A \sin \left(k_{y}\right) \sigma_{x}$ and $h_{12}\left(k_{x}\right)=-B\left(1+e^{-i k_{x}}\right) \sigma_{z}-i A(1-$ $\left.e^{-i k_{x}}\right) \sigma_{y}$. The eigenenergies and eigenvectors at the time-reversal invariant momenta can be obtained analytically. In Fig. $7(\mathrm{a})$ the zero-gap line in the $\left(M_{1}, M_{2}\right)$ plane at each time-reversal invariant momentum and the parities of the occupied bands are shown. When the zero-gap line is crossed, there occurs a band inverting. By combining all the zero-gap lines, the phase diagram in Fig. 5 is recovered. Any phase in the phase diagram can be reached by band inverting starting from a trivial insulator. Thus $\tilde{\delta_{i}}=\delta_{i} \delta_{i}^{\text {trivial }}$ records the number of bands inverting from a trivial insulator. $\tilde{\delta}_{i}=-1$ means an odd number of bands inverting. Then the topological property can be analyzed correctly with $\tilde{\delta}_{i}$.

For the general case without inversion symmetry, the topological property of the $2 \mathrm{D}$ weak TI can be understood from the Berry phase of one spin subsystem, since the 2D weak TI is closely related to $1 \mathrm{D}$ topological phase. The Berry phase defined with $k_{y}\left(k_{x}\right)$ at fixed $k_{x}\left(k_{y}\right)$ can be calculated. The symmetry which protects the 1D topological phase is broken except at specific $k_{x}\left(k_{y}\right)$. If there are two $k_{x}\left(k_{y}\right)$ at which the Berry phase is $\pi$, which means the edge states exist and have two crossings in the presence of an $x(y)$-directed boundary, the system is a $2 \mathrm{D}$ weak TI.

The above analysis is based on the system with spin $s_{z}$ conservation. However, the result is applicable to any time-reversal invariant insulators. Next we study the case of the 
spin-up and -down subsystems coupled by Rashba spin-orbit coupling, which preserve the time-reversal invariant symmetry,

$$
\begin{aligned}
H_{R}= & -\sum_{i, \hat{x}} \operatorname{sgn}(\hat{x}) i \lambda_{R} \Psi_{i}^{\dagger} s_{y} \otimes I \Psi_{i+\hat{x}} \\
& +\sum_{i, \hat{y}} \operatorname{sgn}(\hat{y}) i \lambda_{R} \Psi_{i}^{\dagger} s_{x} \otimes I \Psi_{i+\hat{y}}
\end{aligned}
$$

Add the above term to the modified version of the Hamiltonian equation (1). For the case of anisotropic $B$-type hoppings, the total Hamiltonian in the momentum space is

$$
\begin{aligned}
H_{T}(\mathbf{k})= & \tilde{M}(\mathbf{k}) I \otimes \sigma_{z}+\tilde{A}\left(k_{x}\right) s_{z} \otimes \sigma_{x}+\tilde{A}\left(k_{y}\right) I \otimes \sigma_{y} \\
& +\tilde{\lambda}_{R}\left(k_{x}\right) s_{y} \otimes I-\tilde{\lambda}_{R}\left(k_{y}\right) s_{x} \otimes I .
\end{aligned}
$$

Its energy spectrum is

$$
\begin{aligned}
{\left[E_{T}(\mathbf{k})\right]^{2}=} & {\left[\tilde{A}\left(k_{x}\right)\right]^{2}+\left\{ \pm \sqrt{\left[\tilde{\lambda}_{R}\left(k_{x}\right)\right]^{2}+\left[\tilde{\lambda}_{R}\left(k_{y}\right)\right]^{2}}\right.} \\
& \left.+\sqrt{[\tilde{M}(\mathbf{k})]^{2}+\left[\tilde{A}\left(k_{y}\right)\right]^{2}}\right\}^{2}
\end{aligned}
$$

with $\tilde{A}\left(k_{x}\right)=2 A \sin \left(k_{x}\right), \quad \tilde{A}\left(k_{y}\right)=2 A \sin \left(k_{y}\right), \quad \tilde{\lambda}_{R}\left(k_{x}\right)=$ $2 \lambda_{R} \sin \left(k_{x}\right)$, and $\tilde{\lambda}_{R}\left(k_{y}\right)=2 \lambda_{R} \sin \left(k_{y}\right)$. As has been known, the Rashba spin-orbit coupling does not break the quantum spin Hall effect when it is small. In the following we show that the weak TI is also robust to it. From Eq. (6), it is noticed that the gap closing is independent of $\lambda_{R}$ for $\lambda_{R}<A$. Since the topological quantum phase transition occurs when the gap closes, the weak TI is not affected by the Rashba spin-orbit coupling in this case. For $\lambda_{R}>A$, the gap of the bulk system vanishes at a critical $\lambda_{R}^{c}$, when the weak TI is broken. The calculated energy spectrum and time-reversal polarization $\delta_{i}$ are consistent with the above analysis. For the case of anisotropic mass, the result is similar.

As has been stated, since the midgap edge states in the 2D weak TI are related to the corresponding 1D boundary modes, it can be destroyed by the disorder. However, under specific conditions, they can be robust as those in the $Z_{2}=1$ quantum spin Hall insulators. It can happen in the region near the boundary between WTI and TI phases. For example, for the case with anisotropic $B$-type hoppings, in the middle of the boundary between the WTI and TI phases, the gaps at the two valleys $k_{x(y)}=0, \pi$ are different. For the case shown in Fig. 8, the gap at $k_{y}=\pi$ is nearly zero, while it is still large at $k_{y}=0$. Considering a narrow strip with its edges along the $y$ direction, due to the finite-size effect, the edge states at $k_{y}=\pi$ are gapped, while those at $k_{y}=0$ persist. Thus a single robust gapless crossing at $k_{y}=0$ is realized in the finite-size gap.

\section{CONCLUSIONS AND DISCUSSIONS}

Dimensional evolution between 1D and 2D topological phases is investigated systematically. The crossover from a 2D TI to its 1D limit shows oscillatory behavior between a $1 \mathrm{D}$ ordinary insulator and a 1D TI. By constructing a 2D topological system from a 1D TI, it is shown that there exists a weak topological phase in 2D time-reversal invariant band insulators. The phase can be realized in anisotropic systems. In the weak phase, the topological invariant $Z_{2}=0$ and the edge states only appear along specific boundaries. Since the edge states are closely related to the boundary states of (a)
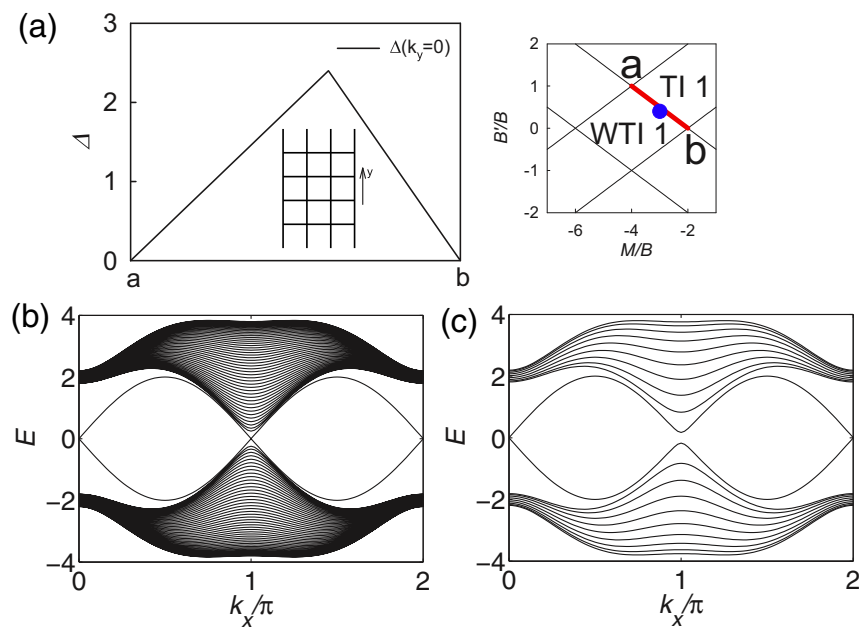

FIG. 8. (Color online) (a) The bulk gap at $k_{y}=0$ with the parameters along the boundary of WTI 1 and TI 1 in the phase diagram (the bulk gap at $k_{y}=\pi$ is all zero). The energy spectrum on a $y$-directed strip with the width (b) $L=50$ and (c) $L=10$. The parameters are $M / B=-3, B^{\prime} / B=0.4$, which is denoted by a blue dot in the right figure of (a) and it is in the WTI 1 phase near the boundary.

the corresponding 1D topological phase, they may be destroyed by disorder and have a symmetry-protecting nature as does the corresponding 1D topological phase. The effect of the Rashba spin-orbit coupling, which preserves timereversal invariant symmetry, but couples the spins, is also studied. These results provide further understanding of 2D time-reversal invariant insulators.

Finally we discuss the relevance of the results to experimental measurements. It is unclear whether the $2 \mathrm{D}$ weak TI materials exist in nature. However, since the anisotropy is important, it should be searched in anisotropic materials. Besides real materials, recently the double-well potential formed by laser light has been developed [48], in which $s$ and $p$-orbital cold atoms can be loaded. It has been shown that a 1D topological model similar to Eq. (2) can be derived from the experimentally realized double-well lattices by dimension reduction [38]. Another experimental platform is the photonic quasicrystals, on which the topological properties have been studied in an engineered way [35]. With the fine tuning of the parameters and the geometries in these experiments, the present results are very possibly realized experimentally.

Note added. Recently, we noticed a recent preprint [49] on closely related topics.

\section{ACKNOWLEDGMENTS}

The authors thank Hua Jiang and Juntao Song for helpful discussions. This work was supported by NSFC under Grants No. 11274032 and No. 11104189, FOK Ying Tung Education Foundation, Program for NCET (H.M.G), and the Research Grant Council of Hong Kong under Grant No. HKU 703713P (S.Q.S). 
[1] J. E. Moore, Nature (London) 464, 194 (2010).

[2] M. Z. Hasan and C. L. Kane, Rev. Mod. Phys. 82, 3045 (2010).

[3] X. L. Qi and S. C. Zhang, Rev. Mod. Phys. 83, 1057 (2011).

[4] S. Q. Shen, Topological Insulators (Springer, Berlin, 2012).

[5] C. L. Kane and E. J. Mele, Phys. Rev. Lett. 95, 146802 (2005).

[6] C. L. Kane and E. J. Mele, Phys. Rev. Lett. 95, 226801 (2005).

[7] L. Fu, C. L. Kane, and E. J. Mele, Phys. Rev. Lett. 98, 106803 (2007).

[8] L. Fu and C. L. Kane, Phys. Rev. B 76, 045302 (2007).

[9] H. Zhang, C.-X. Liu, X.-L. Qi, X. Dai, Z. Fang, and S. C. Zhang, Nat. Phys. 5, 438 (2009).

[10] D. Hsieh, D. Qian, L. Wray, Y. Xia, Y. S. Hor, R. J. Cava, and M. Z. Hasan, Nature (London) 452, 970 (2008).

[11] Y. Xia, D. Qian, D. Hsieh, L. Wray, A. Pal, H. Lin, A. Bansil, D. Grauer, Y. S. Hor, R. J. Cava, and M. Z. Hasan, Nat. Phys. 5, 398 (2009).

[12] H. Lin, L. A. Wray, Y. Xia, S. Xu, S. Jia, R. J. Cava, A. Bansil, and M. Z. Hasan, Nat. Mater. 9, 546 (2010).

[13] Y. L. Chen, J. G. Analytis, J. H. Chu, Z. K. Liu, S. K. Mo, X. L. Qi, H. J. Zhang, D. H. Lu, X. Dai, Z. Fang, S. C. Zhang, I. R. Fisher, Z. Hussain, and Z. X. Shen, Science 325, 178 (2009).

[14] B. A. Bernevig, T. L. Hughes, and S.-C. Zhang, Science 314, 1757 (2006).

[15] M. Konig, S. Wiedmann, C. Brune, A. Roth, H. Buhmann, L. W. Molenkamp, X. L. Qi, and S. C. Zhang, Science 318, 766 (2007).

[16] I. Knez, R.-R. Du, and G. Sullivan, Phys. Rev. Lett. 107, 136603 (2011).

[17] C. X. Liu, H. J. Zhang, B. H. Yan, X. L. Qi, T. Frauenheim, X. Dai, Z. Fang, and S. C. Zhang, Phys. Rev. B 81, 041307 (2010).

[18] H. Z. Lu, W. Y. Shan, W. Yao, Q. Niu, and S. Q. Shen, Phys. Rev. B 81, 115407 (2010).

[19] Y. Zhang, K. He, C. Z. Chang, C. L. Song, L. L. Wang, X. Chen, J. F. Jia, Z. Fang, X. Dai, W. Y. Shan, S. Q. Shen, Q. Niu, X. L. Qi, S. C. Zhang, X. C. Ma, and Q. K. Xue, Nat. Phys. 6, 584 (2010).

[20] P. Cheng, C. L. Song, T. Zhang, Y. Y. Zhang, Y. L. Wang, J. F. Jia, J. Wang, Y. Y. Wang, B. F. Zhu, X. Chen, X. C. Ma, K. He, L. L. Wang, X. Dai, Z. Fang, X. C. Xie, X. L. Qi, C. X. Liu, S. C. Zhang, and Q. K. Xue, Phys. Rev. Lett. 105, 076801 (2010).

[21] R. Yu et al., Science 329, 61 (2010).

[22] C. Z. Chang et al., Science 340, 167 (2013).

[23] J. Wang, B. Lian, H. J. Zhang, Y. Xu, and S. C. Zhang, Phys. Rev. Lett. 111, 136801 (2013).

[24] H. Jiang, Z. H. Qiao, H. W. Liu, and Q. Niu, Phys. Rev. B 85, 045445 (2012).
[25] G. Yang, J. W. Liu, L. Fu, W. H. Duan, and C. X. Liu, Phys. Rev. B 89, 085312 (2014).

[26] B. Yan, L. Müchler, and C. Felser, Phys. Rev. Lett. 109, 116406 (2012).

[27] P. Z. Tang, B. H. Yan, W. D. Cao, S. C. Wu, C. Felser, and W. H. Duan, arXiv:1307.8054.

[28] R. S. K. Mong, J. H. Bardarson, and J. E. Moore, Phys. Rev. Lett. 108, 076804 (2012).

[29] H. Jiang, H. W. Liu, J. Feng, Q. F. Sun, and X. C. Xie, Phys. Rev. Lett. 112, 176601 (2014).

[30] T. Das and A. V. Balatsky, Nat. Commun. 4, 1972 (2013).

[31] L. J. Lang, X. M. Cai, and S. Chen, Phys. Rev. Lett. 108, 220401 (2012).

[32] H. M. Guo and S. Q. Shen, Phys. Rev. B 84, 195107 (2011).

[33] H. M. Guo, S. Q. Shen, and S. P. Feng, Phys. Rev. B 86, 085124 (2012).

[34] H. M. Guo, Phys. Rev. A 86, 055604 (2012).

[35] Y. E. Kraus, Y. Lahini, Z. Ringel, M. Verbin, and O. Zilberberg, Phys. Rev. Lett. 109, 106402 (2012).

[36] M. Atala, M. Aidelsburger, J. T. Barreiro, D. Abanin, T. Kitagawa, E. Demler, and I. Bloch, Nat. Phys. 9, 795 (2013).

[37] W. Tan, Y. Sun, H. Chen, and S.-Q. Shen, Sci. Rep. 4, 3842 (2014).

[38] X. P. Li, E. H. Zhao, and W. V. Liu, Nat. Commun. 4, 1523 (2013).

[39] S.-Q. Shen, W.-Y. Shan, and H.-Z. Lu, SPIN 01, 33 (2011).

[40] A. P. Schnyder, S. Ryu, A. Furusaki, and A. W. W. Ludwig, Phys. Rev. B 78, 195125 (2008).

[41] D. Sticlet, L. Seabra, F. Pollmann, and J. Cayssol, Phys. Rev. B 89, 115430 (2014).

[42] I. M. Shem, T. L. Hughes, J. T. Song, and E. Prodan, Phys. Rev. Lett. 113, 046802 (2014).

[43] J. T. Song and E. Prodan, Phys. Rev. B 89, 224203 (2014).

[44] R. Resta, Rev. Mod. Phys. 66, 899 (1994).

[45] D. Xiao, M. C. Chang, and Q. Niu, Rev. Mod. Phys. 82, 1959 (2010).

[46] B. Zhou, H. Z. Lu, R. L. Chu, S. Q. Shen, and Q. Niu, Phys. Rev. Lett. 101, 246807 (2008).

[47] T. Fukui, K. I. Imura, and Y. Hatsugai, J. Phys. Soc. Jpn. 82, 073708 (2013).

[48] J. Sebby-Strabley, M. Anderlini, P. S. Jessen, and J. V. Porto, Phys. Rev. A 73, 033605 (2006).

[49] Y. Yoshimura, K.-I. Imura, T. Fukui, and Y. Hatsugai, arXiv:1405.4842. 